Página inicial: 137 - Página final: 151

TIPO DE ARTÍCULO: de Investigación

\title{
COMPETENCIAS DOCENTES PARA LA ENSEÑANZA DE LA METODOLOGÍA DE LA INVESTIGACIÓN Y LA EVALUACIÓN DE TRABAJOS DE GRADO Y TESIS DOCTORALES EN ADMINISTRACIÓN ${ }^{6}$
}

\author{
THE TEACHING COMPETENCES FOR THE INSTRUCTION OF RESEARCH AND THE \\ EVALUATION OF RESEARCH PAPERS AND DOCTORAL THESES IN MANAGEMENT.
}

Recibido: Junio 2014 Revisado: Septiembre 2014 Aceptado: Octubre 30 de 2014

Por: Iván Dario Toro Jaramillo, ${ }^{1}$ Juan Guillermo Saldarriaga Ríos, ${ }^{2}$ Mario León Restrepo, ${ }^{3}$ Jormaris Martinez Gómez, ${ }^{4}$ Oscar Arias. ${ }^{5}$

\section{RESUMEN:}

Este artículo aborda un tema de especial relevancia en la dinámica académica actual y en el proceso de formación de los estudiantes de pre y postgrado, pues se relaciona directamente con los procesos de enseñanza y evaluación. Por ello este texto aborda dos procesos relacionados directamente con el quehacer investigativo, dado que se centra en la enseñanza de la metodología de la investigación y en la evaluación de trabajos de grado y tesis doctorales y en las habilidades y competencias que deben desarrollar los docentes para realizar estas actividades.

\section{PALABRAS CLAVE:}

Competencias docentes, metodología de la investigación, evaluación.

\section{ABSTRACT:}

This article discusses a topic of particular relevance in the current academic dynamics and in the process of training of undergraduate and graduate students, as it is directly related to the teaching and evaluation processes. That is why this text addresses these two processes directly related to research activities, given that it focuses on the teaching of research methodology and evaluation of research papers and doctoral theses, as well as in the skills and competencies that teachers must develop for carrying out these activities.

\section{KEY WORDS:}

Teaching Competencies, Research Methodology, and Evaluation.

\footnotetext{
${ }^{1} \mathrm{PhD}$ en Filosofia y PhD en Teología. Coordinado de la Maestría en Ciencias y el Doctorado en Administración en la Universidad de Medellín. Medellín, Colombia. Contacto: idtoro@udem.edu.co, itorojar@gmail.com

${ }^{2}$ Magíster en Psicología, Asistente Dirección de Investigaciones y Postgrados Universidad de San Buenaventura. Medellin, Colombia. Contacto: jusal@une.net.co, juan.saldarriaga@usbmed.edu.co .

${ }^{3}$ Magister en gestión del talento, Universidad de Manizales, directos programa de administración de empresas, fundación universitaria Luis Amigó. Medellín Colombia, contacto: mrestrepo@funlam.edu.co

${ }^{4} \mathrm{PhD}$ en Psicología, Universidad Maimónides, Profesora de Tiempo Completo Fundación Universitaria Luis Amigó. Medellin, Colombia. Contacto: Jormaris@gmail.com, jcastrillon@funlam.edu.co

${ }^{5}$ Magister en Ciencias de la Administración, Institución Universitaria de Envigado. Envigado, Antioquia, Colombia. Contacto: oarias@correo.iue.edu.co
} 


\section{Introducción}

El presente artículo es producto de la primera fase de la investigación "Desarrollo de competencias docentes para la enseñanza de la metodología de la investigación y la formación de evaluadores de trabajos de grado y tesis doctorales en posgrados de administración. Propuesta de un sistema para el desarrollo de competencias docentes en posgrados. Un estudio comparado entre Colombia y Brasil", desarrollado por algunas de las instituciones que pertenecen a la red de investigadores, grupos de investigación y posgrados en Administración.

Esta investigación se basa en el supuesto de que los programas de Administración han enfocado sus esfuerzos en la profundización de la disciplina más que en una reflexión pedagógica; es decir, han centrado sus esfuerzos en "qué" se enseña, más que en "para qué o por qué» se enseña. De ahí que lo importante es el plan de estudios y los contenidos más que las competencias necesarias de los docentes, sobre todo en el caso de las competencias del docente para la enseñanza de la metodología de la investigación. También se observa de manera empírica que no se tiene claro cómo hacer la formación de los evaluadores de trabajos de grado y de tesis doctorales, ya que en la práctica por el solo hecho de tener el título de doctorado se piensa que es suficiente para ser evaluador, inclusive si la línea de investigación y aspectos disciplinares del evaluador no forman parte de las características del trabajo que está siendo evaluado.

Por tanto, la investigación que da origen a este artículo centra sus esfuerzos en comprender aquellos vacíos de conocimiento en el ámbito investigativo con respecto a los programas de posgrado en Administración, como es el desarrollo de competencias docentes para la enseñanza de la metodología de la investigación y la formación de evaluadores de trabajos de grado y tesis doctorales.

\section{Metodologia.}

La investigación es de tipo cualitativo, su población son los docentes de posgrados de Administración de Brasil y Colombia y se desarrolla en cuatro etapas:

La primera, se refiere a la construcción de un estado del arte en relación con lo que se ha escrito sobre el problema de investigación; la segunda, comprende el trabajo de campo, tanto en Brasil como en Colombia. La tercera etapa, de sistematización, consiste en el estudio comparado entre las realidades de cada uno de los países, a partir de la misión de trabajo y la misión de estudio que permitan analizar y organizar los datos. La cuarta etapa es la de difusión de los resultados.

Durante el trabajo de campo previsto en los tres objetivos específicos, utilizaremos las siguientes técnicas de investigación: entrevistas, análisis de contenido y grupos focales con actores del sistema educativo, que puedan dar cuenta del estado actual y de las perspectivas futuras de la formación de los administradores, con el fin de complementar la información recolectada a partir de la revisión de la literatura (estado del arte) y con la eventual aplicación de un instrumento objetivo. 
La sistematización, como tercera etapa de la investigación, prevé la organización de los datos obtenidos en la etapa anterior. Para esto será utilizado el análisis de contenido a partir de las categorías de análisis, con la consiguiente tabulación y cruce de los datos.

\section{Resultados.}

\section{Aproximación A La Temática Objeto De Estudio}

La formación de docentes en posgrados en Administración, tanto en Colombia como en Brasil, está basada especialmente en la experiencia que tiene el docente. Pese a que sea evidente la necesidad que existe de tener docentes formados con un direccionamiento hacia el desarrollo de competencias, especialmente en seminarios que son distintivos en niveles de maestría y doctorado, como es la enseñanza de la investigación. Aun asi, hoy en dia se evidencia un referencial metodológico muy pobre en estos temas, sobre todo si hablamos de la construcción de competencias necesarias para futuros profesores e investigadores.

La experiencia y la prospección exploratoria, realizada en Programas de Administración tanto en Colombia como en Brasil, muestran que en general hay un largo camino por recorrer para alcanzar un concepto de formación que sea adecuado y reconocido; este hecho se evidencia en la demanda de formación de futuros profesores de posgrados que tenemos en Colombia, ya que, actualmente, existe una demostrada escasez de Programas de Maestrias y Doctorados que incluyan el desarrollo de competencias investigativas, pues muchos de los programas ofrecidos son MBA, en los cuales el componente investigativo es superficial.

El desafio para la educación en Programas de Administración en Posgrados de Colombia está en capitalizar los esfuerzos de investigadores brasileros y colombianos para abordar proyectos de investigación que respondan a sus contextos, en vista de la oportunidad abierta por ambos gobiernos para el desarrollo de investigaciones que permitan analizar y reflexionar, en este caso, sobre el desarrollo de competencias docentes para la enseñanza y la formación de evaluadores.

El desarrollo de competencias para la enseñanza en Programas de Posgrados en Administración en Colombia tiene aspectos parecidos a los del Brasil, en cuanto a la institucionalización de los Programas de investigación y los Programas de profundización en Colombia (según señala el art. 24 y 25 del Decreto No. 1295 de 20 de abril de 2010 del Ministerio de Educación Nacional de Colombia). Los Programas de maestría de profundización, podrian definirse como equivalentes a las maestrías profesionalizantes en Brasil, y los programas de maestría investigativa y programas de doctorado en Administración podrían definirse como equivalentes a los programas académicos brasileros. Los referentes de educación colombianos no priorizan la dimensión de formación de competencias del docente en la enseñanza, así como ocurre en el contexto brasilero. 
Según la información suministrada por el profesor Anielson Barbosa da Silva, de la Universidad Federal de Paraiba, el desarrollo institucional, a nivel de regulación y evaluación en posgrados, tuvo en Brasil un avance sustancial desde la década de 2000, con el direccionamiento más evidente de cursos de maestría y doctorado para la formación de investigadores, como primera prioridad, y de profesores para la educación a nivel de posgrado como segunda prioridad. Esta realidad se basó en la proposición de programas de posgrados basados en el concepto de "académico", para usar un término de referencia regulatorio brasileño más cercano.

Sin embargo, debido al desarrollo de la estructura académica general de Brasil, la formación de posgrado con enfoque profesionalizante alcanzó una organización más amplia a partir de la década del 2000, especialmente con el desarrollo de los cursos de maestría profesionalizante. Mediante esta estructura, en la última década se inició en Brasil el desarrollo de la postgraduación en Administración direccionada en términos de formación para la investigación científica, para el ejercicio profesional y en gran parte para el ejercicio de docencia, aunque la vocación por la formación de los profesores aún no se ha logrado plenamente.

Por su parte en Colombia, la mayor parte de postrgados en Administración, especialmente en el nivel de Maestría tienen un enfoque profesionalizante, siendo pocos los programas orientados al desarrollo de las competencias investigativas y la formación de investigadores. En el nivel de formación doctoral, según el SNIES, existen en el país 9 programas, en los cuales, por el nivel, la pretensión es la formación de investigadores, pero no así el desarrollo de competencias para la enseñanza de la investigación y para la evaluación de trabajos de grado y tesis doctorales.

\section{El Concepto De Formación}

La formación, en cuanto concepto de la disciplina pedagógica parece en principio un abstracto, lo cual puede explicarse porque dentro de dicha disciplina es un concepto relativamente nuevo. Según Vanegas (2004), sus raíces se encuentran el en término "forma" y desde la pedagogía se le han dado muchos usos según el entender de quien lo utiliza. Así las cosas, las diferentes corrientes pedagógicas entienden la formación como algo diferente para cada una de ellas, pero con el rasgo común de que va más allá de la educación o de la transmisión de conocimientos e implica necesariamente la participación activa del sujeto.

La formación entonces es un acto de intencionalidad concreto en el que las subjetividades de quien educa o forma y de quien es educado o formado se encuentran con el fin de producir un salto cualitativo en la construcción del conocimiento. Por ello, el sujeto que se forma no es pasivo, es un agente activo y protagonista de su propio proceso. Así las cosas, las competencias de los profesores para la formación van más allá del aspecto académico y tienen estrecha relación con el ser.

La Administración como campo de conocimiento y como práctica no ha dejado de ser mecanicista. Tanto la práctica como la enseñanza han sido y siguen siendo instrumentales. Según Klisberg (1995) esto lo que hace es mirar al hombre dentro 
de la organización desde una óptica puramente mecanicista y lo ubica solamente como un medio o una herramienta que sirve a los fines productivos de la organización y que puede ser descartado en cualquier momento. El hombre es sólo un recurso para la producción, postulados con los que concuerda Aktouf (1998).

Los libros de texto y best sellers introducen en el escenario administrativo una serie de pasos que deben seguirse de manera ordenada, coherente y lógica para asegurar el éxito de la gestión y desarrollar una estrategia de recursos humanos cuya pretensión es la tendencia a la generalización y la aplicabilidad en cualquier organización. Esto es lo que muchos profesores entienden como la formación ideal para sus estudiantes y es de esta concepción que se nutren las "recetas" que los estudiantes de pregrado y postgrado aprenden en las aulas de clase y que pretenden llevar a las organizaciones. Quienes beben de estas concepciones tratan de aplicar pasos estrictos a la hora de administrar o gerenciar la organización y generan acciones que se quedan en el plano de la administración instrumental.

Marín Idárraga (2006), insiste en la necesidad de que la Administración deje de lado la naturaleza instrumental que la ha acompañado a lo largo de su desarrollo y que ubica al ser humano en un plano de simple recurso y recalca la necesidad de reconocer al sujeto humano dentro de la administración, desde una perspectiva compleja. Hacia alli tiende el concepto de formación que desde el escenario pedagógico se desea alcanzar.

Competencias Docentes Para La Enseñanza De La Metodología De La Investigación

En el nivel universitario de posgrados, quienes enseñan generalmente asumen los roles de docentes, asesores y tutores. Así mismo, evaluadores, quienes asumen la responsabilidad de evaluar y calificar los trabajos de grado de maestría y las tesis doctorales, en algún momento asumen o han asumido los roles anteriormente mencionados. En algunas ocasiones, cada uno de ellos puede asumir otro rol paralelamente. Acorde a esto, se considera que quién ha sido asesor de trabajos de grado de maestría o tutor de tesis doctoral, está en la capacidad de ser evaluador de las mismas, pues ha desarrollado y adquirido la suficiente experticia tanto académica como personal, para hacerlo.

Desde este punto de vista y teniendo en cuenta que por lo general los docentes, asesores, tutores o evaluadores en posgrado, no tienen formación en pedagogia, es necesario realizar un acercamiento a cuáles son las competencias que deben tener quienes se desempeñen en estos roles, con el objetivo de mejorar la calidad en la educación y en segunda instancia, las evaluaciones de desempeño que se realizan a quienes se desempeñan en ello, sin dejar de reconocer que el nivel de formación de estas personas (maestría y doctorado) les confiere por derecho propio una experticia propia del área de conocimiento a la cual pertenezcan y en cuanto al desarrollo de investigaciones, ésta última siempre y cuando sus estudios hayan sido investigativos y no profesionalizantes. 
En este sentido, y de acuerdo a los roles que pueden asumirse, existe un vacío en la literatura frente a la definición de docente desde el posgrado, así como las competencias que el mismo debiera poseer. La mayoria de las definiciones se han creado para los niveles de primaria, secundaria y pregrado, siendo necesario adaptarlas al nivel posgrado.

Valcárcel (2003, pág.169) define la tutoría como un "período de instrucción realizado por un tutor con el objetivo de revisar y discutir los materiales y temas presentados en las clases".

Para Jiménez (1996, pág.14-15), el concepto de formador se refiere a toda persona que de alguna manera tiene relación con la formación desde la Óptica de la responsabilidad de la concepción o de la realización de la misma. Comprende un grupo heterogéneo de personas, con profesiones muy distintas, difícil de discernir. La distinta procedencia, su formación inicial, su concepción teórica y práctica de la vida y del trabajo, la diversidad de las situaciones en las que tienen que operar (formación profesional reglada, no reglada), los niveles y modalidades de formación, las especialidades, los materiales que deben emplear o diseñar, la diversidad de los grupos destinatarios, no hacen sino añadir complejidad a una "profesión" nueva y no perfilada en la mayor parte de sus competencias.

Esta definición, si bien no utiliza el término docente, se relaciona directamente con los roles de docente, asesor y tutor a los cuáles nos referimos en este artículo y plantea, respecto a las competencias, la falta de claridad.

Dupont y Reis (1991) en Jiménez (1996), plantean que existen unos tipos de profesionales, entre los que se encuentran los a) los especialistas en la cuestión pedagógica y concepción de la formación, b) los especialistas y técnicos en la gestión de la formación, c) los enseñantes y los profesores (enseñanzas técnicas y profesionales), d) los formadores a jornada completa, e) los formadores a jornada parcial y los formadores ocasionales. Según Jiménez (1996, pág.17), los formadores a jornada completa han puesto sus conocimientos y su experiencia no al servicio de la empresa, sino de la docencia. Implica un cambio de actividad. (...) Se detecta en ellos deficiencias pedagógicas que se ponen de manifiesto a la hora de definir objetivos, elaborar materiales, explicar conceptos o procedimientos, secuencializar contenidos, utilizar medios y recursos de enseñanza, evaluar o establecer relaciones con alumnos, aprendices o adultos en formación.

Indudablemente, esta tipificación hace referencia a los docentes, en cualquier nivel de formación y permite ilustrar gran parte de las características de los actuales docentes de los posgrados, pues al ser tan especializada su experticia en un área particular de conocimiento, es alli donde se encuentra su mayor fortaleza y desarrollo de competencias, más que en el ejercicio de la formación de personas desde la docencia. En esta medida, los docentes, asesores, tutores y evaluadores son formados, en ocasiones para ser formadores, desde un área específica de conocimiento, sin tener esto sustento desde las competencias que se espera adquieran para este rol. 
Al realizar una búsqueda sobre literatura o investigaciones realizadas acerca de las competencias docentes para la enseñanza de la metodología de la investigación y la evaluación de trabajos de grado en posgrados, se evidencia poca productividad en esta área. En general, las investigaciones están centradas en la evaluación docente en pregrado (pocas en postgrado), en la percepción de los estudiantes sobre el ejercicio docente y en los valores que debe desarrollar un docente para su ejercicio. Esto permite evidenciar que la educación posgradual ha estado más enfocada, desde la investigación, en generar conocimiento nuevo, que en cuestionarse la formación y las competencias que deben tener aquellos que enseñanza en este nivel de educación. Se podría inferir que, la condición de tener un título de doctorado o maestria, provee automáticamente las competencias y habilidades para enseñar y evaluar trabajos de grado y tesis a nivel de maestría y doctorado. De igual manera, a menos que se cuente con la suficiente experiencia investigativa que pueda ser homologada, para ser docente o evaluador de cada uno de estos niveles, se debe tener al menos el mismo nivel de formación. Sin embargo, este tema está directamente relacionado con la calidad de la educación que se ofrece y los valores que se promueven a través de la misma.

En la literatura científica se encuentran múltiples y diversas definiciones de competencias, lo que para algunos autores genera vacíos conceptuales. Altarejos (1998) plantea que una competencia es un concepto que hace referencia a la capacidad o habilidad para la resolución de dificultades que características del trabajo que se realice. Por su parte, para Bar (1999) existe la competencia especificadora, la cual se refiere a la capacidad para la aplicación de un cúmulo de conocimientos esenciales en la comprensión de un determinado tipo de sujetos, instituciones o fenómenos y procesos, con un dominio mayor de los contenidos y metodologías de las diferentes disciplinas.

Durante varios años, diferentes autores han propuesto una serie de competencias que deben desarrollar los docentes. Entre ellos se encuentra Braslavsky (1998) quien plantea que los docentes deben desarrollar las competencias a) pedagógicodidáctico y b) político-institucional (ambas relacionadas con la resolución de problemas), c) productiva e d) interactiva (estructurales) y e) especificadora (especialización y orientación de la práctica profesional).

A partir de un estudio de investigación, Valcárcel (2003) propone que dentro del perfil de los docentes universitarios (definiendo perfil profesional (pág. 54) como el "conjunto de capacidades y competencias que identifican la formación de una persona, para asumir en condiciones óptimas las responsabilidades propias del desarrollo de funciones y tareas de una determinada profesión"), deben estar las siguientes competencias:

- Cognitivas: directamente relacionadas con la función de docente de una disciplina determinada, llevando a un extenso conocimiento en las esferas disciplinar propia y pedagógica.

- Meta-cognitivas: le permiten al docente, de manera sistemática, la revisión y mejoramiento de su enseñanza a partir de la autocrítica y la reflexión. 
- Comunicativas: se relacionan directamente con el adecuado uso de un lenguaje científico así como de las diferentes formas de registrarlo.

- Gerenciales: se encuentran ligadas con la enseñanza y sus recursos y la gestión eficiente de los mismos, en diferentes entornos y ambientes de aprendizaje.

- Sociales: favorecen, entre otros, la realización de acciones de cooperación, liderazgo y trabajo en equipo, promoviendo el desarrollo profesional tanto de sí mismo como la formación de los estudiantes.

- Afectivas: estas competencias garantizan actitudes, conductas y motivaciones que alimentan la responsabilidad y el compromiso de la docencia con los objetivos de formación.

Siguiendo esta línea, en el 2005, Pérez, propone las siguientes competencias minimas generales y específicas para los profesionales en educación:

Cuadro 1. Competencias generales y específicas según Pérez

\begin{tabular}{|c|c|}
\hline COMPEIIENCIAS GENERALES & COMPEIENCIAS ESTECCIRICAS \\
\hline C. Comunicacionales & $\begin{array}{l}\text { 1.- Mejora en los procesos de comunicación } \\
\text { 2.- Fomento de actividades de dinamización la formación del } \\
\text { profesorado a nivel europeo } \\
\text { 3.- Sensibilización del profesorado en el análicis, revisión y mejora } \\
\text { de su propia formación } \\
\text { 4.- Establecimiento de foros de reflexión sobre acciones formativas } \\
\text { abiertos a la participación de compañeros europeos }\end{array}$ \\
\hline C. Organizativa & $\begin{array}{l}\text { 1.-Transferencia de aprendizajes en la formación permanente y } \\
\text { aplicación de recursos innovadones } \\
\text { 2.- Interpretación de la realidad docente y establecimiento de los } \\
\text { oportunos procesos de mejora } \\
\text { 3.- Mejora de la convivencia universitaria e institucional }\end{array}$ \\
\hline C. de Liderazgo pedagógico & $\begin{array}{l}\text { 1.- Relación con el profesorado de ámbitos cercanos y ampliación } \\
\text { de horizontes en las relaciones internacionales } \\
\text { 2.-Trabajo en equipo y superación de fronteras geográficas } \\
\text { 3.- Impulso de la dimensión europea y apoyo de los procesos de } \\
\text { comunicación con otras lenguas }\end{array}$ \\
\hline C. Cientificas & $\begin{array}{l}\text { 1.- Formación en contenidos científicos, didácticos y metodológicos } \\
\text { 2.- Realización de proyectos innovadores propios de la universidad } \\
\text { 4.- Desarrollo del pensamiento empirico ante las nuevas realidades } \\
\text { 5.- Impulso de la innovación y en la investigación científica }\end{array}$ \\
\hline C. de Evaluación y Control & $\begin{array}{l}\text { 1.- Evaluación permanente de los procesos de formación del } \\
\text { profesorado } \\
\text { 2.- Establecimiento y diseño de formaciones específicas con el fin de } \\
\text { superar los puntos débiles y potenciar los fuertes }\end{array}$ \\
\hline
\end{tabular}

Fuente: Pérez (2005. P. 3) 
Por su parte Hisch (2005) realizó una investigación para construir una escala de actitudes sobre ética profesional para lo cual evaluó a 131 personas entre las cuales se encontraban profesionales, docentes universitarios, estudiantes de posgrado y población general. A partir de las respuestas, la autora construyó 18 rasgos que clasificó en 5 tipos de competencias las cuales son las cognitivas (preparación $\mathrm{y}$ formación continua e innovación), b) técnico-organizativas (conocimiento y competencia técnica y eficiencia y eficacia), c) sociales (compañerismo y buenas relaciones, comunicación, saber trabajar en equipo, dedicación y ser trabajador), d) éticas (responsabilidad) y e) afectivo-emocionales (identificarse con la profesión y capacidad emocional). Finalmente para la elaboración del instrumento sólo tuvo en cuenta las competencias cognitivas, sociales, éticas y afectivo emocionales.

Posteriormente Tejada, en el 2009, propuso que en la formación de formadores, se deben tener presentes las siguientes competencias para los formadores: teóricas o conceptuales, psicopedagógicas y metodológicas y sociales.

Por su parte, en el 2011, Serna y Luna a partir de un análisis de 135 docentes de posgrado, identificaron y jerarquizaron valores, competencias y rasgos que, de acuerdo a los mismos docentes, eran los más importantes para tener en cuenta en su ejercicio. Para las competencias utilizaron la misma clasificación propuesta por Hirsch (2005). Estas autoras (Serna \& Luna, 2011) identificaron que los valores más importantes en su orden son la integridad, el profesionalismo, el respeto, la competencia en la materia y la solidaridad y los de menor importancia la congruencia, el ser demócrata y la colaboración. Así mismo, las competencias cognitivas y éticas son las más valoradas y las competencias sociales (compañerismo y relaciones y comunicación e interés en la formación de los alumnos) las menos valoradas. Sin embargo, la valoración de la importancia de las competencias depende del área de conocimiento de los docentes. En estos llama la atención como los docentes que participaron en el estudio, dan mayor valor a la integridad que a la competencia en la materia y menor valor a la colaboración, pues en algunas ocasiones en la práctica docente en posgrados, los trabajos de grado y las tesis doctorales de los estudiantes están a cargo de una determinada persona quién es la que acompaña todo el proceso, y estos trabajos generalmente son socializados en los grupos generando competitividad, lo que puede dificultar la generación de un ambiente de colaboración, dado que cada uno está interesado en llevar a feliz término y de la mejor manera posible su proyecto de investigación.

\section{El Concepto De Evaluación}

Al igual que la formación, la evaluación se puede entender de diferentes maneras. Pareciera que esta es una de las características del saber pedagógico, pues la multiplicidad de vertientes teóricas y posiciones epistemológicas hacen imposible que los conceptos ligados a la pedagogía puedan ser abordados desde una perspectiva universalmente aceptada. Sin embargo, existen algunas características que pueden ser evidenciadas en cualquier concepto de evaluación que se tenga, una de ellas es la perspectiva desde la que se realiza, ya sea netamente cuantitativa, de control y 
medición del producto o la cualitativa y de proceso; en cualquiera de los casos lo que se pretende es realizar una medición y calificación de un trabajo determinado.

Stufflebeam y Shinkfield (1995. P. 19), citados por Mora (2004; 2) señalan que la evaluación es el enjuiciamiento sistemático de la validez o mérito de un objeto. Si es un proceso de enjuiciamiento se supone ya que quien evalúa posee los conocimientos, técnicas y competencias para emitir un juicio real acerca de la calidad de lo evaluado y lo que parece existir en el proceso de evaluación de los trabajos de grado y tesis doctorales no es que los evaluadores posean las técnicas y competencias para hacerlo, pues usualmente se eligen con base en sus conocimientos y este juicio que en muchas ocasiones es parcializado y mediado por las posiciones teóricas, epistemológicas y paradigmas investigativos es tomado como real, válido y cierto por la comunidad académica.

La evaluación educativa como proceso siempre ha existido, pero ha ido cambiando al igual que se dan los cambios y procesos sociales. Según De la Garza (2004; 809) "la evaluación educativa tiene su origen en los primeros años del siglo XX en, Estados Unidos. Está influida por las ideas de progreso, por la administración científica y por la ideología de la eficiencia social". En este sentido, es una evaluación de carácter cuantitativo y objetivo y que se ha impuesto de manera generalizada en las diferentes ciencias y disciplinas, la Administración no escapa a ello, y más cuando ha sido una potenciadora de su aparición.

La evaluación educativa supone que existen algunos acuerdos y consensos previos en lo referente a lo que se quiere evaluar y lo que es considerado generalmente como "bueno" o "malo" de acuerdo con el propósito de las acciones educativas. En el caso de los ejercicios investigativos en ocasiones parece que este consenso no existe, puesto que quien evalúa los trabajos lo hace desde su propio observador, con la carga de sus posiciones teóricas y epistemológicas, lo que le concede a este tipo de evaluación una gran carga de subjetividad, aunque lo que se pretenda sea precisamente lo contrario, es decir, la objetividad. La evaluación, entonces, tiene un carácter ideológico y político que en su proceso técnico e instrumental obedece a una lógica de control.

Para De La Garza (2004; 809) “el mismo carácter instrumental de la evaluación contraviene el sentido desinteresado de la obtención de nuevo conocimiento que subyace a la lógica de la ciencia. Lo cierto es que, en un sentido amplio, el propósito de los proyectos de evaluación es la obtención de conocimiento, si bien se busca que éste sea útil para la toma de decisiones". Esto implica que la evaluación, especialmente aquella que se realiza a los trabajos investigativos, obedece a la lógica de la construcción del conocimiento, pero de aquél que es considerado válido y relevante en un contexto determinado y se encuentra cargada de subjetividad en el marco de una lógica objetiva, para la que muchos evaluadores no se encuentran formados ni preparados. 
El proceso de formación profesional de Pregrado e incluso de Maestría y Doctorado los estudiantes tienen desde un vago acercamiento a los procesos de investigación en los niveles inferiores hasta una fuerte especialización en los niveles superiores. Este conocimiento de la metodología de la investigación, en conjunto con la experiencia adquirida en el campo investigativo mediante la formulación y desarrollo de proyectos de investigación ayudan a que los investigadores desarrollen unas habilidades y competencias para juzgar el trabajo propio y el de otros investigadores. Estas competencias son puestas a prueba al momento de leer, evaluar y calificar un trabajo investigativo, especialmente cuando dicha evaluación se hace en un marco institucional y su funcionalidad es conceder o no un título académico.

Tradicionalmente tanto las universidades como las revistas científicas las han recurrido a investigadores expertos a la hora de evaluar los trabajos investigativos que se publican o que conducen al título académico. En este proceso, la pretendida objetividad radica en el proceso evaluativo ciego - ciego, en el que quien evalúa no conoce al sujeto evaluado y el evaluado desconoce quien realiza la evaluación. Pero se han olvidado que la "objetividad" va más allá del proceso y aunque no quiera reconocerse, se encuentra en el sujeto, por tanto la objetividad es, en esencia, una subjetividad autorregulada.

¿Esta autorregulación se aprende en el transcurso de la formación profesional o está dada por la experiencia investigativa del evaluador y su posición ética ante la construcción del conocimiento? Al tratar de dar respuesta a esta pregunta desde el punto de vista teórico se encuentra una radical ausencia de teoría o de trabajos investigativos que aborden este objeto de estudio. Podría recurrirse a la Filosofía de la Ciencia, a la Epistemología y a la Ética en procura de un acercamiento, pero entendiendo (como usualmente se hace en las universidades) el aspecto de la evaluación de los trabajos de investigación como un asunto de orden instrumental y técnico, dicho análisis desbordaría el objeto.

En la literatura es dificil encontrar una reflexión acerca de la evaluación de los trabajos de grados y más difícil aún hallar investigaciones que aborden esta temática. Lo que se encuentra es una serie de recetas emanadas desde las universidades como modelos propios para evaluar los ejercicios investigativos, circunscribiendo la evaluación a formatos y procedimientos preestablecidos que casi cualquier persona con formación y conocimientos básicos en investigación puede realizar y en los casos más elevados de construcción de conocimiento (como el Doctorado), quienes son expertos en una temática y han alcanzado este mismo título pueden hacerlo.

No quedan claras entonces las competencias que debe tener un buen evaluador de trabajos de grado y tesis doctorales, pues en una revisión simple de los protocolos que tienen las diferentes universidades se da cuenta de que este es un aspecto de carácter contextual, que los formatos diseñados para realizar la evaluación corresponden a los intereses institucionales y no se deja ver de manera explícita cuáles son dichas competencias. Implícitamente se podría hablar de conocimientos teóricos acerca del tema a evaluar, conocimientos (que pueden ser teóricos o 
prácticos) de la metodología de la investigación (cuantitativa o cualitativa según el trabajo a evaluar), comprensión lectora, habilidades para la redacción y una gran dosis de irreverencia, autoconfianza, sentido de grandiosidad y tendencia a la descalificación.

Estas características son necesarias para que quien evalúa un trabajo pueda detectar en él los aspectos dignos de ser resaltados, pero también aquellos que no son lo suficientemente fuertes, que no contribuyen de manera significativa a cualificar el trabajo, que aparecen como teórica y metodológicamente incoherentes y que son necesarios de revisar y rehacer por parte del autor.

Estas competencias pues, se adquieren en el proceso de formación como investigador, pero también en el proceso de convertirse en persona. Son características que no sólo brinda la academia o la experiencia investigativa, sino que se construyen con el ser humano mismo y que le permiten ponerse en el lugar de quien juzga para contribuir a cualificar el trabajo y la experiencia subjetiva del otro en cuanto ser humano que investiga.

\section{Conclusiones.}

Es usual que en el escenario de la Administración, como en el de cualquier disciplina se hable de las competencias que se requieren de los profesionales que egresan de las universidades. Los profesores (por formación o por desempeño) no son ajenos a estas exigencias y lo que se espera de ellos es que tengan introyectadas determinadas competencias para ejercer su labor y que al mismo tiempo puedan ayudar a sus estudiantes a desarrollar algunas de ellas.

En el caso de los profesores de Metodología de Investigación y evaluadores de trabajos de grado y tesis doctorales, se supone que quienes han tenido un recorrido importante en investigación poseen, de suyo, las competencias para desarrollar estas actividades. Es un supuesto ampliamente generalizado que los investigadores expertos son también "buenos" profesores de investigación y "buenos" lectores y evaluadores de trabajos investigativos. Al examinar el currículo de los programas de Pregrado, Postgrado, Maestria y Doctorado, se puede observar fácilmente que en ninguno de los niveles de formación se forma o siquiera se enseña a los estudiantes los aspectos básicos que les permitan desempeñar con eficiencia y eficacia el rol de profesores de Metodología de la Investigación y mucho menos adquirir las competencias para considerarse adecuados evaluadores de trabajos de grado y tesis doctorales.

En la actualidad, tanto en Colombia como en Brasil, los programas de postrgrado en Administración, especialmente las Maestrías, tienen una orientación profesionalizante, orientada al "hacer" y a la formación de gerentes hábiles para la toma de decisiones organizacionales pero no para el desempeño académico. En el mejor de los casos en estos programas se fortalecen algunas competencias docentes y se forman profesores bastante competentes en algunas áreas administrativas, 
pero no se forman investigadores ni profesores de investigación y mucho menos lectores y evaluadores de trabajos de grado y tesis doctorales.

En la formación doctoral, tanto en Colombia como en Brasil, los programas de Administración pretenden desarrollar competencias investigativas en los estudiantes, lo que se hace con base en ejercicios y procesos investigativos sólidos, en la reflexión teórica y en el análisis disciplinar. Pero estos procesos no son suficientes para desarrollar las competencias docentes en el campo de la investigación y en lo que hace referencia a la lectura y evaluación de trabajos de grado y tesis, puede decirse que en estos programas no hay ningún tipo de formación. Esta es una competencia desarrollada de manera autónoma por los investigadores y basada en su propia experiencia investigativa. 


\section{Referencias Bibliográfica.}

Aktouf, O. (1998). La administración: entre tradición y renovación. Cali. Artes Gráficas Univalle.

Altarejos, F. (1998). La docencia como profesión asistencial. En F.A. Altrejos, J.A. Ibañez-Martín, J.A. Jordán \& G. Jover, Ética docente. Barceloona: Ariel, 21-50.

Bar, G. (1999). Perfil y competencias del docente en el contexto institucional educativo. Recuperado de http://www.oei.es/de/gb.htm

De La Garza, E (2004). La evaluación educativa. Revista Mexicana de Investigación Educativa. 9(23). 807-816.

Braslavsky, C. (1999). Bases, orientaciones y criterios para el diseño de programas de formación de profesores. Revista Iberoamericana de Educación 19: eneroabril. Recuperado de http://www.rieoei.org/oeivirt/rie19a01.htm

Hirsch, A. (Mayo, 2005). Construcción de una escala de actitudes sobre ética profesional. Redie. Revistaa Electrónica de Investigación Educativa, 7(1), 1-14. Recuperado de http://redie.uabc.mx/vol7no1/contenido-hirsch.html.

Jiménez, B. (1996). Los formadores. Educar, 20, 13-27. Recuperado de http://ddd. uab.cat/pub/educar/0211819Xn20p13.pdf

Klisberg, B. (1995). El pensamiento organizativo: de los dogmas al nuevo paradigma gerencial. Buenos Aires.

Luna, E., \& Serna, A. (Julio-diciembre, 2011). Valores y competencias para el ejercicio de la docencia de posgrado. Sinéctica, 37. Recuperado de http://www. sinectica.iteso.mx/index.php?cur=37\&art=37_04

Marín, D. (2006). El sujeto humano en la administración: una mirada crítica. En: Cuadernos de Administración. N N 32. Bogotá. Julio - Diciembre de 2006. p. $135-156$.

Mora, A. (2004). La evaluación educativa: concepto, períodos y modelos. Revista electrónica actualidades investigativas en Educación. 4(2).

Pérez, M. (febrero, 2005). La formación permanente del profesorado ante los nuevos retos del sistema educativo universitario. Revista Electrónica Interuniversitaria de Formación del Profesorado, 8(1), 1-4. Recuperado de http:/ /www.redalyc.org/ articulo.oa?id=217017146007

Tejada, J. (2009). Competencias docentes. Profesorado. Revista de curriculum y formación del profesorado, 13(2), 1-15. Recuperado de http://www.ugr. es/ recfpro/rev132COL2.pdf 
Valcárcel Cases, M. (2003). La preparación del profesorado universitario español para la convergencia Europea en Educación Superior. Informe Final. Recuperado de http: / / www.ugr.es/ filosofia/recursos/mejora/2006/3.tutorias/preparacionprofesorado.pdf

Vanegas, M. (2004). El concepto pedagógico de formación en el universo semántico de la educación. Revista Educación, 28(2). 13-28. Recuperado de http://www. redalyc.org/articulo.oa?id $=44028202$

\title{
Nota:
}

\begin{abstract}
${ }^{6}$ Este artículo es producto de la investigación "Desarrollo de competencias docentes para la enseñanza de la metodología de la investigación y la formación de evaluadores de trabajos de grado y tesis doctorales en posgrados de Administración. Un estudio comparado entre Colombia y Brasil", cuyo objetivo es: analizar desde una perspectiva critica el desarrollo de las competencias del docente para la enseñanza de la metodología de la investigación, y la formación de evaluadores de trabajos de grado y de tesis doctorales en posgrados de Administración, mediante la realización de estudios comparados entre Brasil y Colombia. La investigación viene siendo desarrollada en el marco de la Red de investigadores, grupos de investigación y postgrados en Administración y es cofinanciada por Colciencias (Colombia) y CAPES (Brasil)
\end{abstract}

Las universidades que participan en la investigación son: Universidad Simón Bolívar, Universidad de Medellín, Universidad de San Buenaventura, Fundación Universitaria Luis Amigó, Institución Universitaria de Envigado y Universidad Federal de Paraiba 